\title{
Experimental investigation of the flow characteristics of low-pressure drop air nozzles working with CFB boilers
}

\author{
Pawet Mirek ${ }^{*}$ \\ Institute of Advanced Energy Technologies, Faculty of Infrastructure and Environment, Czestochowa \\ University of Technology, Dabrowskiego 69, 42-201 Częstochowa, Poland
}

\begin{abstract}
The paper presents the most important flow characteristics of low-pressure drop primary air nozzles used in circulating fluidized bed boilers. The role of an air nozzle pressure drop, pressure fluctuation, as well as air jet penetration length, orifice outlet jet kinetic energy and aerodynamic drag force of the gas outlet jet have been discussed. For the purpose of cold model studies the Lagisza $966 \mathrm{MW}_{\text {th }}$ supercritical CFB boiler operating at the company TAURON Wytwarzanie SA, Poland has been chosen as the reference facility. In the experimental tests, three types of primary air nozzles with comparable static pressure drop and varied aerodynamic drag force have been tested. The experiments clearly showed that the pressure drop cannot be the only parameter characterizing the operation of the primary air nozzle and the air jet penetration length is determined primarily by the orifice outlet jet kinetic energy and not by the outlet jet velocity.
\end{abstract}

\section{Flow characteristics of air nozzles}

Flow characteristics of air nozzles working with circulating fluidized bed (CFB) boilers can be regarded as a set of parameters determining the ability of nozzles to ensure homogeneous and stable fluidization in the cross-section of the combustion chamber, as well as to supply the primary air in a way that prevents:

- erosion of neighboring nozzles and boiler lining walls,

- backflow of a gas-solid mixture into the air plenum chamber,

- erosive self-destruction of the nozzle,

- the formation of "dead zones" on an air distributor, where the loose material is not subjected to the process of intensive mixing.

Optimal nozzle design should also ensure an easy horizontal flow of solids on the grid surface resulting from a transport a loose material moving in the internal (combustion chamber) and external (boiler return system) circulation loop.

\footnotetext{
*Corresponding author: pmirek@neo.pl
} 


\subsection{Grid pressure drop. Fluctuations of static pressure}

Providing homogeneous and stable fluidization in the whole cross-section of the combustion chamber is only possible when the resistance of the air distributor for the vertical (upward) and the horizontal gas flow is determined by the relationship $\Delta p_{d} \geq 0.3 \Delta p_{b}$, while for the gas flowing downwardly by the relationship $\Delta p_{d} \geq 0.1 \Delta p_{b}$. For large-scale CFB boilers, it is obligatory to satisfy the following requirement [1]

$$
\Delta p_{d} \geq \text { from } 2500 \mathrm{~Pa} \text { to } 3600 \mathrm{~Pa}
$$

For the fluidized beds of a considerable size $\left(H_{m f} / d_{t}<0.5\right)$, the relationship between the pressure drop on the air distributor and the bed material can be determined by the following expression [2]

$$
\frac{\Delta p_{d}}{\Delta p_{b}} \geq \exp \left(-3.8 \frac{H_{m f}}{d_{t}}\right)
$$

In Polish CFB boilers, the ranges of pressure drops for minimum and maximum boiler loads are $5.1 \mathrm{kPa}$ and $8.9 \mathrm{kPa}$ respectively. In the case of low-pressure drop air nozzles, the static pressure fluctuations caused by the turbulent mixing of a loose material have a significant influence on the nozzle operation. In order to avoid the migration of solids into the nozzle, it is recommended that the length of the outlet channels should be within the range of 0.05 to $0.1 \mathrm{~m} \mathrm{[3]}$.

\subsection{Air jet penetration length}

Although there are no recommendations in the literature regarding the nozzle's outlet velocity of the air, it is assumed to determine a parameter dependent on it, which is the air jet penetration length. The value of this parameter should not be greater than the distance between the outlet of the nozzle and the furnace lining surface or a neighboring nozzle. According to the formula proposed by Merry [4] and taking into account Karri's recommendations [5], the air jet penetration length (downwardly directed) can be estimated based on the following expression

$$
L=7.875 d_{w}\left(\frac{\rho_{g w} u_{w}^{2}}{\rho_{s}\left(1-\varepsilon_{m f}\right) g d_{32}}\right)^{0.4}\left(\frac{\rho_{g b}}{\rho_{s}}\right)^{0.2}\left(\frac{d_{32}}{d_{w}}\right)^{0.2}
$$

Since the air nozzles equipped with the vertically downward arms are not used in Polish CFB boilers, the equation (3) should be regarded as the approximate formula.

\subsection{Orifice outlet jet kinetic energy}

The orifice outlet jet kinetic energy, just as the air jet penetration length, determines the mode of air supply by the nozzle to the boiler's combustion chamber. As follows from $[6,7]$, in the case, when:

- $\quad E_{k}>W_{w}$ - the air is supplied to the combustion chamber via narrow gas jets, causing limited mixing of the loose material in the area of the air distributor (risk of channeling), abrasion of solid particles and a strong erosion of the nozzles and boiler's linings walls, 
- $\quad E_{k}<<W_{w}$ - solids weeping can occur; in many regions of the grid, the loose material remains motionless (the gas jet will not contribute much to the bed stirring [6]), which creates a risk of occurring sinters

A very useful factor that measures the stirring effect of the air distributor is the stirring factor being a function of the kinetic energy of the air outlet jet from the nozzle and the pressure drop on the bed material, given by the following expression [7]

$$
\alpha=\frac{\rho_{f} u_{w}^{2}}{2 \Delta p_{b}}
$$

When $\alpha>1$, the air jet is supplied with the very narrow gas channels that prevent effective mixing of bed material and cause attrition of particles and erosion of bed internals [7]. For $\alpha \ll 1$, the mixing of solids occurs mainly as a result of the interaction and eruption of gas bubbles.

\subsection{Aerodynamic drag force}

With respect to the primary air nozzles, the aerodynamic drag force is a relative measure of the resistance of the nozzle to the backflow of a bed material. Assuming that the drag coefficient is determined by the relationship proposed by Haider and Levenspiel [8]

$$
C_{D}=\frac{24}{R e_{p}}\left[1+\left(8,1716 e^{-4,0655 \varphi_{s}}\right) R e_{p}^{0,0964+0,5565 \varphi_{s}}\right]+\frac{73,69\left(e^{-5,0748 \varphi_{s}}\right) R e_{p}}{R e_{p}+5,378 e^{6,2122 \varphi_{s}}}
$$

the aerodynamic drag force can be calculated based on the following general equation

$$
F_{A}=C_{D} S_{d} \frac{\rho_{f} \cdot u_{w}^{2}}{2}
$$

The aerodynamic drag force and, as a result, the resistance of the nozzle to the backflow of solids increases with the increase of the air outlet velocity from the nozzle. However, it should be noted that the maximum value of this parameter depends on the permissible jet penetration length resulting from the height of the nozzles on the air distributor and the grid pitch.

\section{Characteristics of the reference facility}

The reference facility to which all analyzes are referenced is the CFB-1300 $466 \mathrm{MW}_{\mathrm{e}}$ supercritical CFB boiler operating at TAURON Wytwarzanie SA - The Lagisza Power Plant, Poland. The air distributor of the boiler is equipped with tuyeres of a comparable pressure drop, whose basic parameters determined during experimental tests are presented in Table 1 . 
Table 1. Summary of basic air nozzles' parameters used on the air distributor of the CFB-1300 $466 \mathrm{MW}_{\mathrm{e}}$ supercritical CFB boiler at $100 \% \mathrm{MCR}$

\begin{tabular}{lccrr}
\hline & & & \\
\hline
\end{tabular}

As can be seen from Table 1, the nozzles have been designed in such a manner that they can be used on the boiler grid interchangeably. Their common and characteristic feature is the manner of supplying the air to the combustion chamber in one plane. It results from the grid pitch and nozzles' mutual placement on the surface of an air distributor.

\section{Experimental test-stands}

Experimental tests of flow characteristics of air nozzles were carried out on two experimental test-stands. The first one, presented in Figure 1, is used to measure the pressure drop, its fluctuations as well as the kinetic energy and aerodynamic drag force of the outflowing air. 


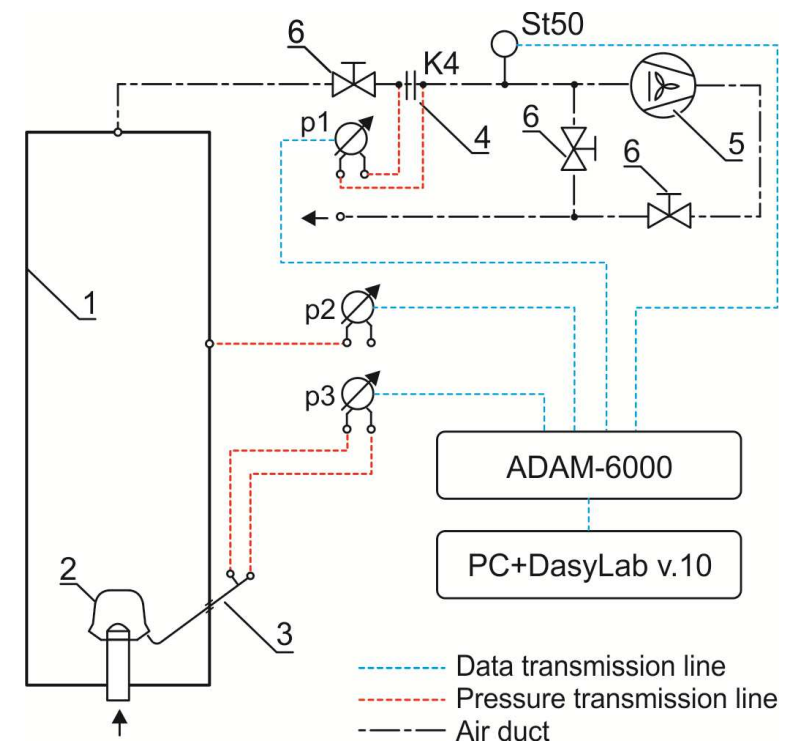

Fig. 1. Schematic diagram of the test-stand used for determination of the pressure drop and the outlet velocity of an air nozzle1 - Partial vacuum chamber, 2 - Air nozzle (1:1 scale), 3 - Prandtl tube, 4 - Measuring orifice plate K4, 5 - Centrifugal industrial fan $\left(5500 \mathrm{~m}^{3} / \mathrm{h}, 20 \mathrm{kPa}\right), 6$ - Control valves, p1, p2, p3 - Digital pressure sensors APR-2000ALW, St50 - Thermal flow meter

The main part of the test-stand is a working chamber 1, in which the tested air nozzle 2 is installed. The air flow through the chamber is supplied by a centrifugal industrial fan 5 with an adjustable rotational speed. The air fan has the following parameters: $\mathrm{Q}_{\mathrm{v}}=5500$ $\mathrm{m}^{3} / \mathrm{h}, \Delta \mathrm{p}=20 \mathrm{kPa}$. The volumetric flow of the air is measured by the orifice plate K4 4 and simultaneously by the thermal flow meter FCI St50. The pressure drop generated by the air nozzle is measured by the $\mathrm{p} 2$ sensor, whereas the air velocity at the outlet opening is measured by the Prandtl tube coupled with the pressure transducer p3. The ambient pressure is measured by using the Label LB-750 electronic type barometer. The data acquisition system consists of the ADAM-6000 measuring cards coupled with a PC and the DasyLab software.

Experimental tests of the nozzle set with the gas-solid mixture have been carried out on the test stand, whose schematic diagram is depicted in Figure 2. 


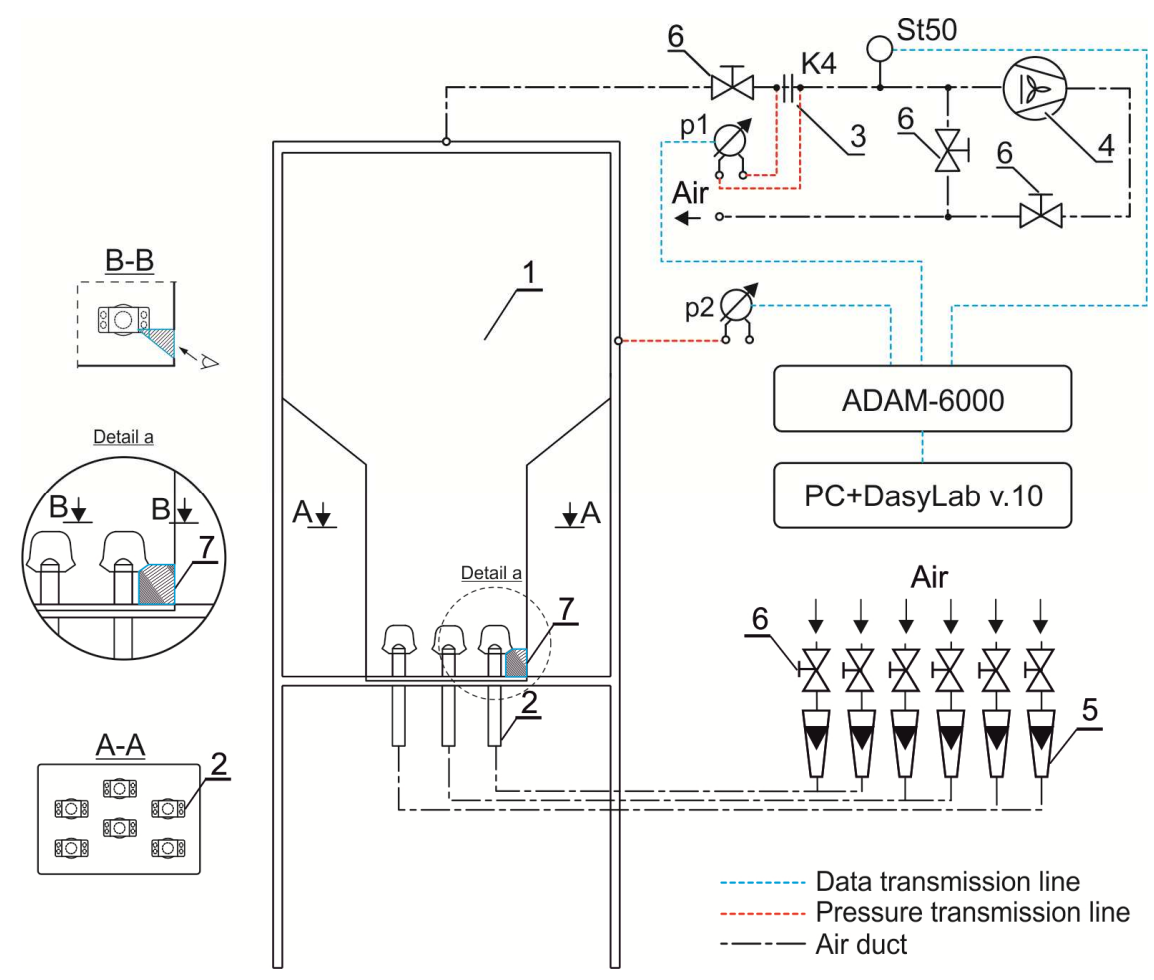

Fig. 2. Cold model test-stand for testing the set of air nozzles with the gas-solid mixture. 1 - test chamber, 2 - air nozzle, 3 - measuring orifice plate K4, 4 - centrifugal industrial fan $\left(5500 \mathrm{~m}^{3} / \mathrm{h}\right.$, $20 \mathrm{kPa}$ ), 5 - rotameters, 6 - control valves, 7 - inspection window, p1, p2 - digital pressure sensors APR-2000ALW, FCI ST50 - thermal flow meter

The main part of the test-stand is a transparent chamber 1 in which six full-scale air nozzles are installed according to the grid pitch typical for the CFB-1300 CFB boiler. The cold model is connected to an industrial fan $4\left(\mathrm{Q}_{\mathrm{v}}=5500 \mathrm{~m}^{3} / \mathrm{h}, \Delta \mathrm{p}=20 \mathrm{kPa}\right)$. The amount of air supplied to each nozzle can be adjusted independently by valves 6 and measured by rotameters 5 . The measurement of the air jet penetration length is carried out with the help of the inspection window 7. Similarly as in the case of the system shown in Fig. 1, the atmospheric pressure is measured by using the Label LB-750 electronic type barometer, while in the acquisition of measurement data the ADAM-6000 measurement cards as well as the DasyLab software are used. As a particulate material, quartz sand particles with a particle size distribution similar to that of the bottom ash occurring in the CFB-1300 supercritical CFB boiler are used in the tests.

\section{Experimental test results}

Figure 3 presents the characteristics of the dimensionless pressure drop and the air outlet velocity measured in laboratory conditions for the tested air nozzles. In the case of pressure characteristics, the results of measurements are referred to the maximum pressure drop registered for the Nozzle 3 supplied by the volumetric air flow equal to $0.04 \mathrm{~m}_{\mathrm{n}}^{3} / \mathrm{s}$. As can be seen from the obtained distributions, the pressure drops (from approx. 1000 to about $3500 \mathrm{~Pa}$ ) for each of the tested nozzles are comparable within the entire range of a thermal 
load of the boiler. However, taking into account the fact that in the case of bubble caps type air nozzles the pressure drop is within the range from 4.7 to $8.9 \mathrm{kPa}$, the results presented in Fig. 3 can be considered relatively small.

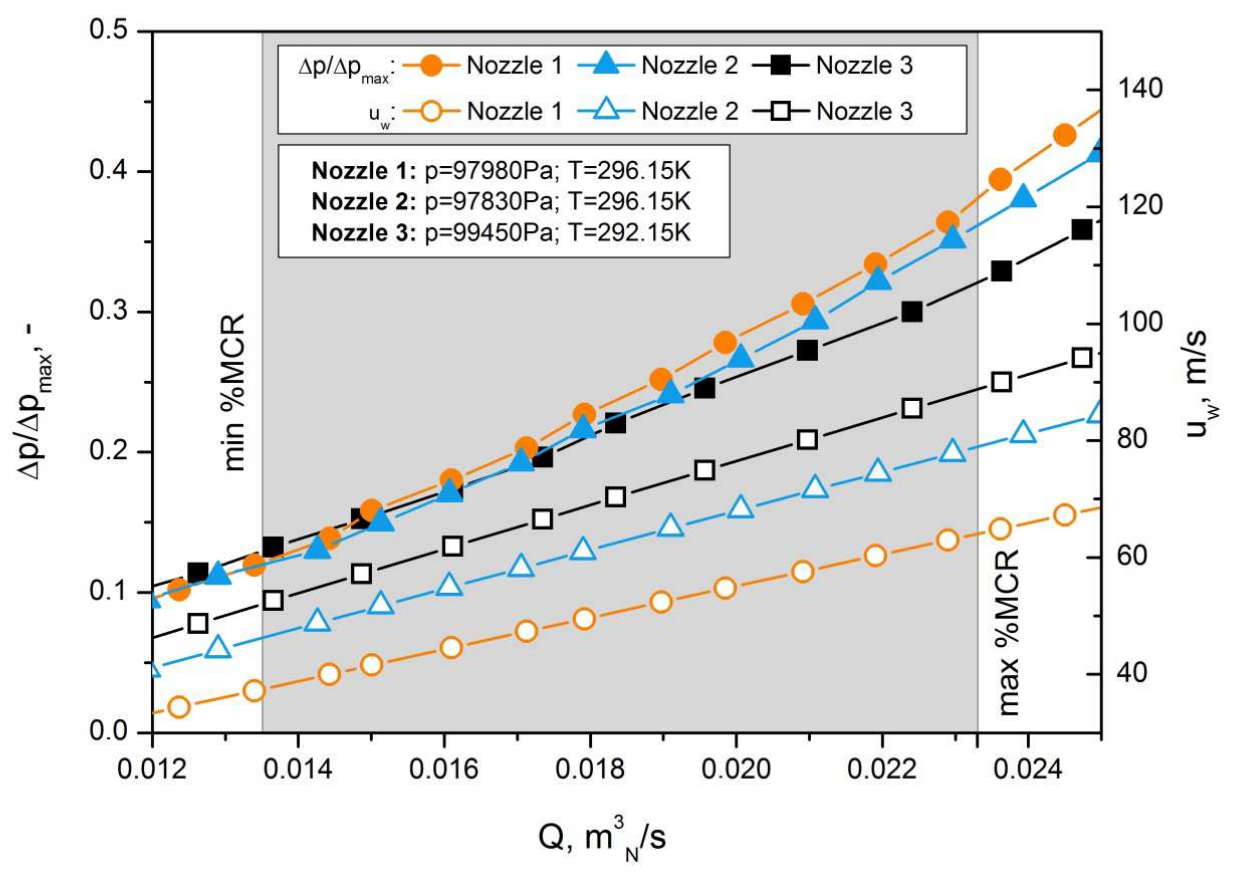

Fig. 3. Comparison of the relative drag characteristics and the orifice outlet velocity for the tested air nozzles. The shaded area corresponds to the operation range of the CFB-1300 CFB boiler

Analyzing the characteristics of the orifice outlet velocity, it should be noted that the highest values of this parameter can be observed for the Nozzle 3 with the smallest crosssection of the outlet opening. In this case, the orifice outlet velocity is less than $90 \mathrm{~m} / \mathrm{s}$. Since the high orifice outlet velocity carries the risk of a strong erosive impact of particles of a loose material, the examination of the nozzles has been supplemented by the studies of the air jet penetration length. Figure 4 depicts the comparison of the characteristics of the air jet penetration length for the tested air nozzles calculated based on the Eq. (3). As follows from the comparison of results presented in Figs. 3 and 4, the kinetic energy of the orifice outlet air jet is an important parameter in the modeling of the air jet penetration length. The largest length of the air jet has been determined for the nozzle with the highest orifice outlet jet kinetic energy, i.e. Nozzle 2. This nozzle is also characterized by the highest stirring factor, promoting the best mixing of solids and keeping the bed well fluidized. The results of the calculations have been verified experimentally for Nozzle 3, showing differences at the level not greater than $20 \%$. 


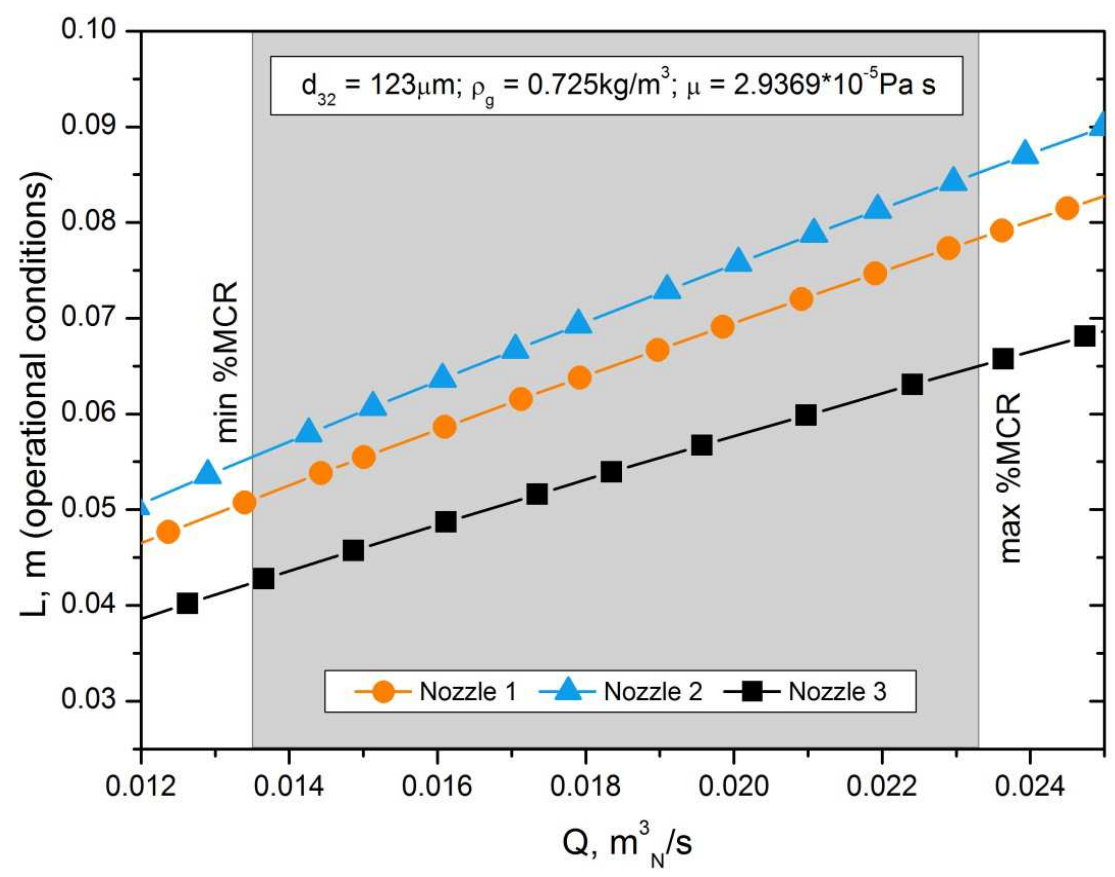

Fig. 4. Comparison of the characteristics of the air jet penetration lengths for the tested air nozzles. The shaded area corresponds to the operation range of the CFB-1300 CFB boiler

Although the air jet penetration length is a parameter that usually determines the degree of erosive impact of the gas-solid outlet stream on the lining surfaces of the boiler or the adjacent nozzle, it may also be a measure of a degree of self-erosive impact of a nozzle. Among the tested nozzles, this tendency has been observed for the Nozzle 3. When the nozzle is supplied with a small volumetric air flow, a local turbulent vortex is generated in the vicinity of the nozzle outlet opening causing an erosive impact of particles on the upper surface of the nozzle outlet arm (see Fig. 5).

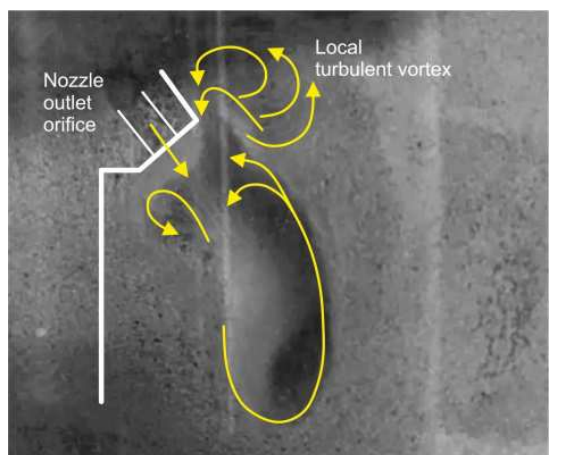

Fig. 5. Image of a self-erosive impact the local turbulent vortex of the Nozzle 3 in the vicinity of the nozzle outlet opening 
From the operational point of view, a very important parameter of an air nozzle is the resistance to the pressure fluctuation that causes backflow of solids into an air plenum chamber. One of the characteristics of this resistance is an aerodynamic drag force of the nozzle. Figure 6 presents the comparison of the aerodynamic drag force characteristics of the tested nozzles. As indicated by the obtained distributions, the aerodynamic drag force increases with the increase of the volumetric air flow (and indirectly, air outflow velocity) and reaches the maximum value for the four-hole air nozzle (i.e. Nozzle 3). The aerodynamic drag force of that nozzle is more than two times larger within the whole range of the CFB-1300 boiler thermal load as compared to the Nozzle 1.

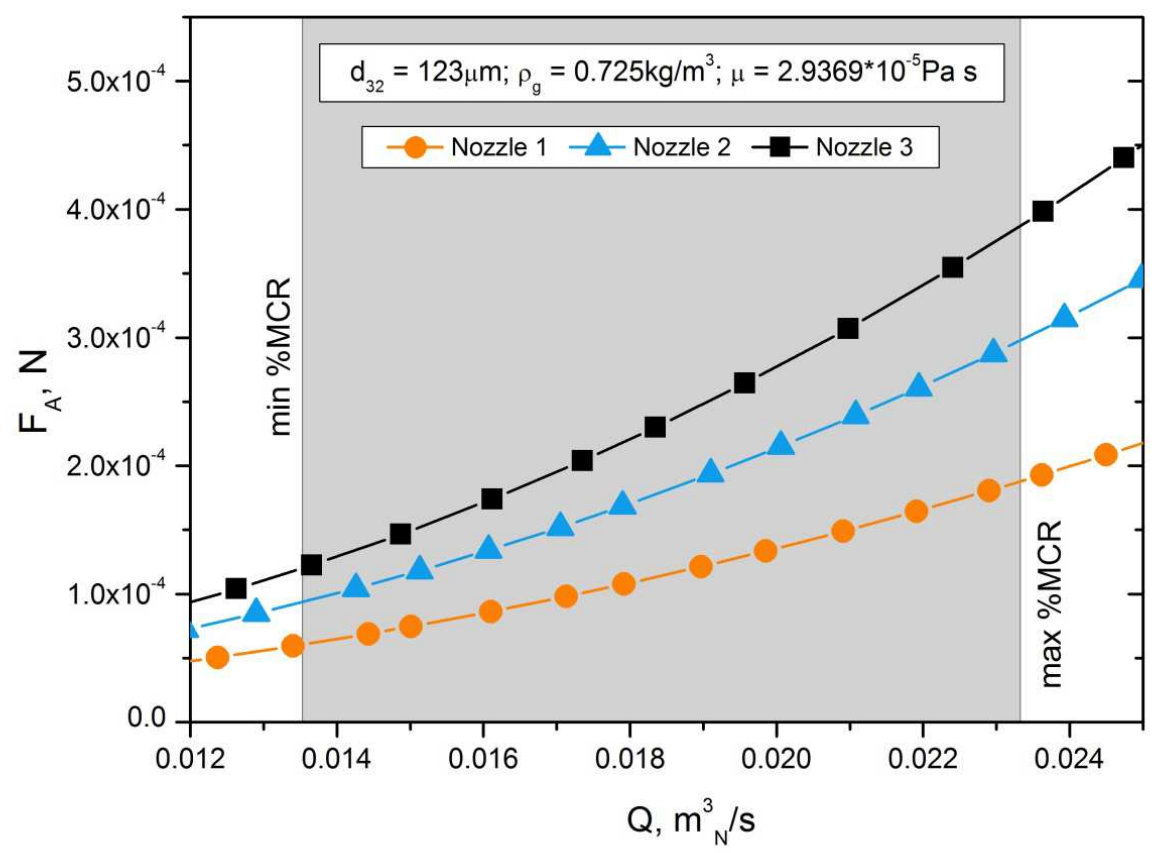

Fig. 6. Comparison of the aerodynamic drag force characteristics of the tested air nozzles. The shaded area corresponds to the operation range of the CFB-1300 CFB boiler

Based on the results of laboratory measurements, it was found that both the Nozzle 2 and the Nozzle 3 are characterized by a high resistance to the backflow of solids. Therefore, the values of $0.8 \cdot 10^{-4}$ and $2.9 \cdot 10^{-4} \mathrm{~N}$, corresponding to the minimum and maximum thermal load of the boiler, should be considered as critical values of the aerodynamic drag force for tuyeres designed for the CFB-1300 CFB boiler. The results of experimental studies have been confirmed during operational tests which ultimately confirmed the high resistance of the Nozzle 2 and Nozzle 3 to the bed material backflow under variable mass load conditions.

\section{Summary}

The flow characteristics of tuyere-type air nozzles working in CFB boilers determine a set of parameters allowing for long-term and failure-free operation of the air distributor, providing homogeneous and stable fluidization in the entire cross-section of the boiler's combustion chamber. The experimental tests carried out on three different designs of air 
nozzles clearly showed that the pressure drop cannot be the only parameter characterizing the operation of these devices. The tested air nozzles had a comparable resistance within the entire range of the supply volumetric air flow revealing completely different orifice outlet velocities, the air jet penetration length as well as the aerodynamic drag force. Laboratory tests also showed that the orifice outlet jet kinetic energy, not the velocity, directly determines the air jet penetration length. The parameter that plays an important role in the reduction of the backflow of solids into the air plenum chamber is the aerodynamic drag force of the nozzle. A value of this force can be changed in a simple way by the size of the cross-section of the nozzle outlets. Taking into consideration the fact that there is a strong relationship between the flow characteristics of the nozzles mentioned above, it is advisable to carry out a careful multicriterial optimization in the process of designing a tuyere-type air nozzle in order to determine the best, from the operational point of view, values of the nozzle's pressure drop, orifice outlet jet kinetic energy as well as aerodynamic drag force. 


\section{Notation}

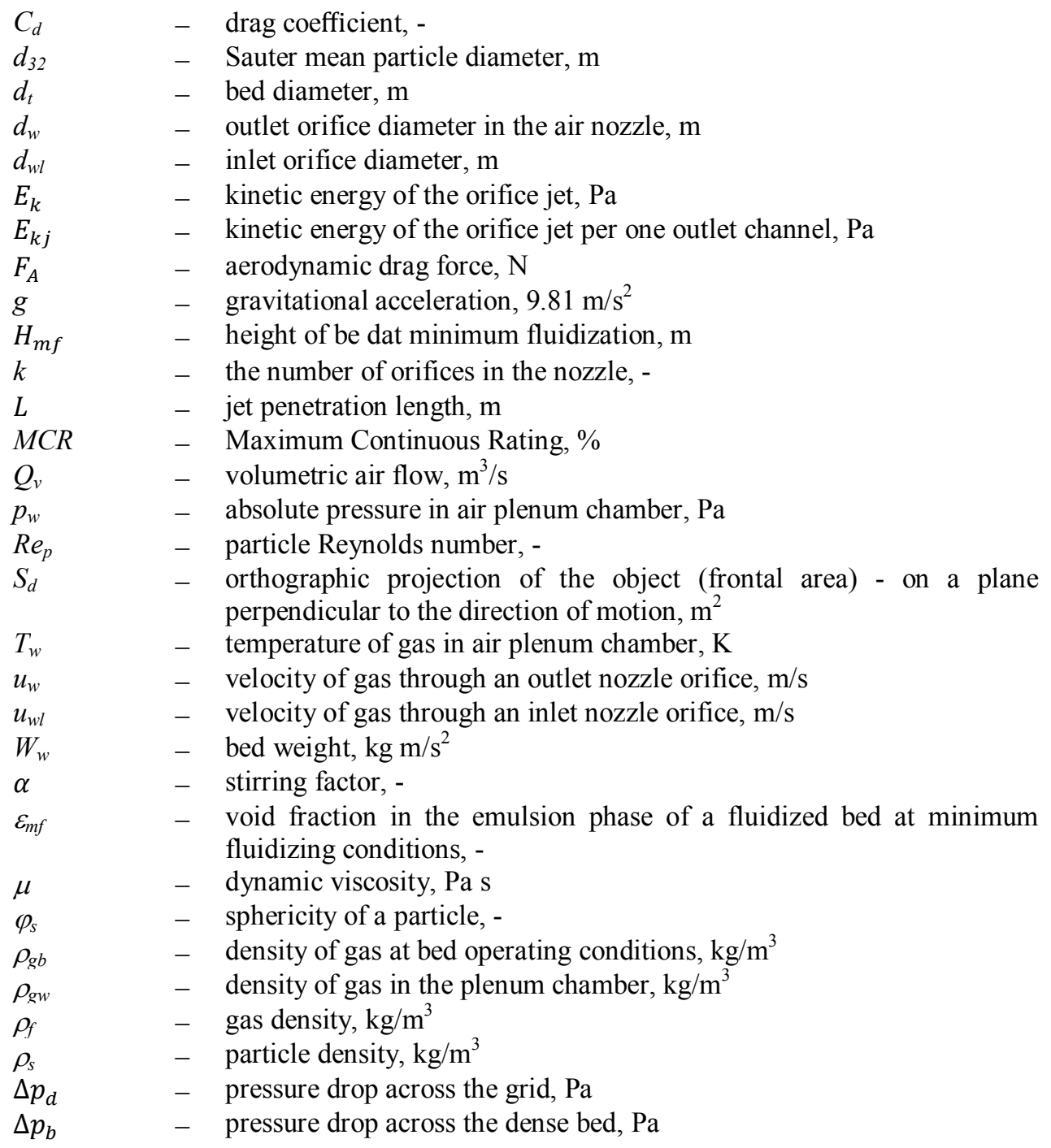

This investigation was financially supported by the fund of statutory research of Czestochowa University of Technology. Project No: BSPB-406-301/11. 


\section{References}

1. S.B. Karri Reddy, J. Werther, Handbook of Fluidization and Fluid-Particle Systems, Wen-Ching Yang: Siemens Westinghouse Power Corporation, Pittsburgh, Pennsylvania, USA, (Marcel Dekker Inc., Chap. 6, 2003)

2. D. Geldart, J. Baeyens, Powder Technol., 42 (1985)

3. J. Werther, Circulating Fluidized Bed Technology VIII, Cen, K., Ed., Int. Academic Publishers, Beijing, (2005)

4. J.M.D. Merry, Trans. Inst. Chem. Engrs, 49:189 (1971)

5. S.B. Karri Reddy, PSRI Research Report No. 60, (1990)

6. S.C. Saxena, D. Sathiyamoorthy, C.V. Sundaram, Transport Processes in Fluidized Bed Reactors, Advances in transport processes, Doraiswamy L.K., Kulkarni B.D., Volume VII, 242, (Wiley Eastern Limited, eds., 1987)

7. D. Kunii, O. Levenspiel, Fluidization Engineering, 2nd ed, (Butterworth-Heinemann, 1991)

8. A. Heider, O. Levenspiel, Powder Technol., 58 (1989) 